\title{
Preparation and characterization of silver nanoparticles in natural polymers using laser ablation
}

\author{
REZA ZAMIRI*, B Z AZMI, HOSSEIN ABBASTABAR AHANGAR ${ }^{\dagger}$, GOLNOOSH ZAMIRI, \\ M SHAHRIL HUSIN and $Z$ A WAHAB \\ Department of Physics, Faculty of Science, Universiti Putra Malaysia, 43400 UPM, Serdang, Selangor, Malaysia \\ ${ }^{\dagger}$ Department of Chemistry, Faculty of Science and Engineering, Najafabad Branch, Islamic Azad University, \\ Najafabad, Iran
}

MS received 30 September 2011; revised 25 October 2011

\begin{abstract}
In this paper we have done a comparative study on efficiency of natural polymers for stabilizing silver nanoparticles (Ag-NPs) prepared by laser ablation technique. The selected polymers are starch (St), gelatin (Gt) and chitosan $(\mathrm{Ct})$. The fabrication process was carried out through ablation of a pure Ag plate by nanosecond Q-switched Nd-Yg pulsed laser ( $\lambda=532 \mathrm{~nm}, 360 \mathrm{~mJ} /$ pulse). The stability of the samples was studied by measuring UV-visible absorption spectra of the samples one month after preparation. The result showed that the formation efficiency of NPs in St were highest and also the prepared NPs in St solution were more stable than other polymers during one month storage.
\end{abstract}

Keywords. Laser ablation; silver nanoparticles; UV-spectroscopy.

\section{Introduction}

Strong research efforts have been devoted in the last decade to develop synthesis of noble metal NPs because of their important applications in many scientific areas. Among noble metal NPs, Ag-NPs show unique chemical, physical and optoelectronic properties (Tsuji et al 2008; Blanco-Andujar et al 2010; Zhang and $\mathrm{Xu}$ 2010) with good potential for use in medical diagnosis, recorded media and optics. However, there is still a big challenge to synthesize stable collides containing Ag-NPs in various sizes and shapes for precise applications. Indeed, metal NPs will tend to agglomerate via precipitation and flocculation due to thermodynamic principle. This problem can be overcome by using chemical species which passivate NPs making their agglomeration difficult. So far, several chemical and physical methods for synthesis of Ag-NPs have been used by researchers (Tolles 1996; Pimpang et al 2008) but laser ablation in liquid is predominant as a nonchemical approach for the production of colloidal NPs. Its remarkable features are simplicity and influence of the surrounding solvent that is different with laser ablation in gas or vacuum (Tsuji et al 2008). A number of important physical and chemical effects of solvent are cooling, confinement and oxidation or reduction (Simakin et al 2001; Tsuji et al 2003, 2008). In addition, stability and particle size of NPs were affected by the presence of adducts in solution (Mafune et al 2001; Bae et al 2002). Laser ablation is carried out in various types of stabilizers such as soluble starch, gelatin and chitosan to prevent agglomeration of NPs (Darroudi et al 2011; Zamiri et al 2011a, b).

In the present paper in continuation of our previous studies (Darroudi et al 2011; Zamiri et al 2011a, b), we compare the efficiency of Ct, Gt and St on the stability of Ag-Nps prepared by laser ablation method. Ag plate was irradiated in aqueous solution of these stabilizers with a pulsed Nd:YAG

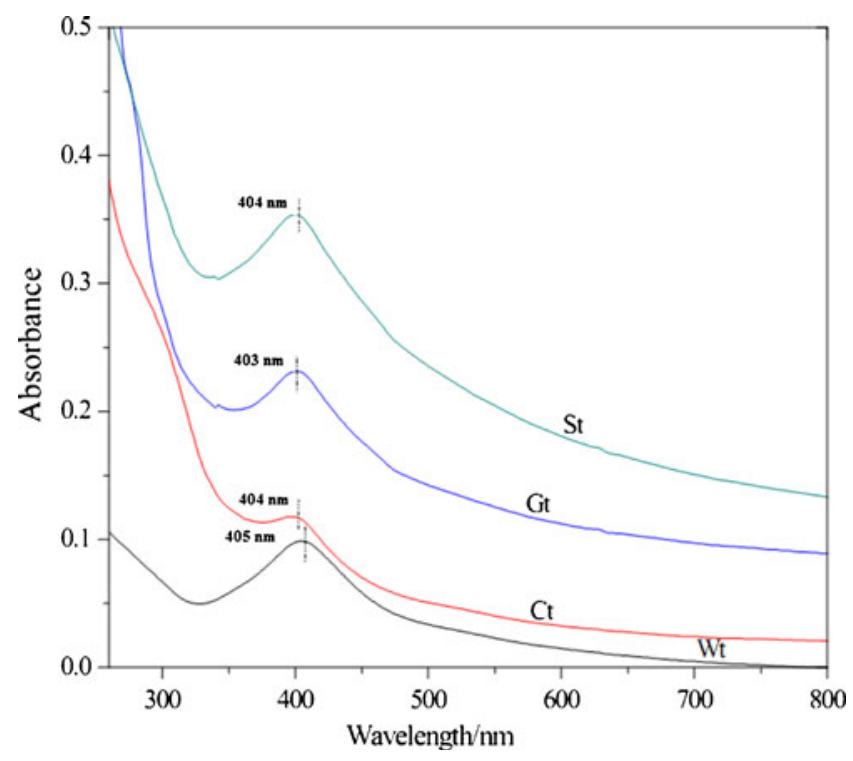

Figure 1. UV-Vis absorption spectra of all samples.

\footnotetext{
*Author for correspondence (azmizak@gmail.com)
} 

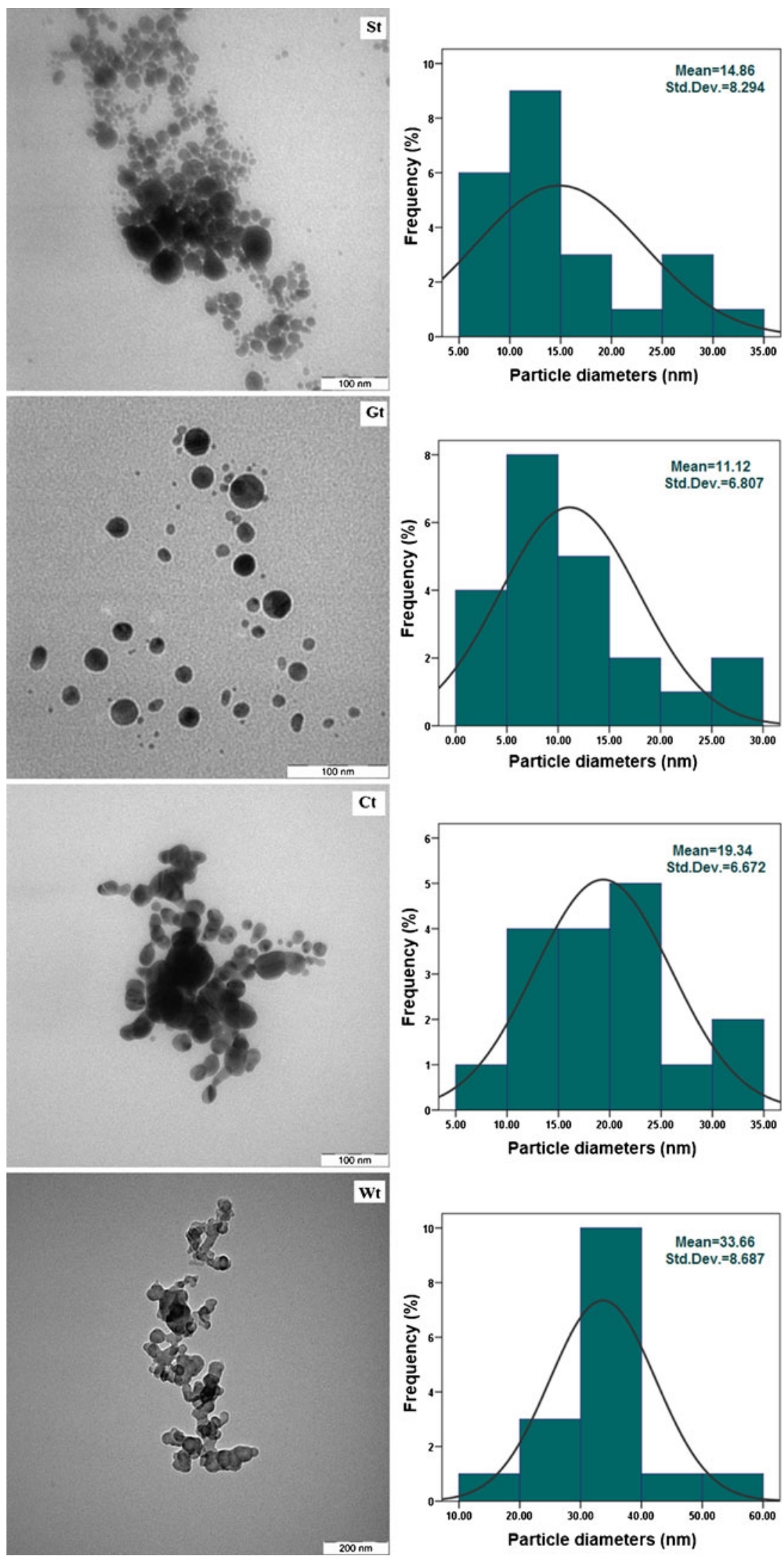

Figure 2. TEM images of all samples. 
laser for same period of time. Ag atoms ejected from the metal surface by the local high temperature and high pressure plasma plumes due to interactions of metal surface and pulsed laser beam. Then, nano-sized Ag-NPs were produced through cooling and condensation of ejected $\mathrm{Ag}$ atoms by solvent (Park et al 2010; Zamiri et al 2011c).

\section{Experimental}

$0.5 \mathrm{~g}$ of $\mathrm{Ct}$, Gt and St were dissolved in $100 \mathrm{ml}$ distilled water (wt) at $60{ }^{\circ} \mathrm{C}$ and stirred for $1 \mathrm{~h}$. An Ag plate (SigmaAldrich, 99.99\% purity) was first washed in ultrasonic bath and then placed around $4 \mathrm{~mm}$ behind the cell wall. The cell was filled with $20 \mathrm{~mL}$ of $\mathrm{Ct}, \mathrm{Gt}$, St and Wt. The metal plate was irradiated by a pulsed Q-switched Nd:YAG laser (Brilliant) with pulse duration of $5 \mathrm{~ns}$ energy, $360 \mathrm{~mJ} /$ pulse and $10 \mathrm{~Hz}$ repetition rate at its lasing wavelength of $1064 \mathrm{~nm}$. The laser beam was focused on target by a lens with a focal length of $250 \mathrm{~mm}$. The solution was stirred magnetically during the ablation process in order to disperse the produced NPs. After synthesis process, prepared Ag-NPs were characterized using UV-visible double beam photo-spectrometer (Shimadzu, UV-1650 ${ }_{\mathrm{PC}}$ ), transmission electron microscopy (TEM, Hitachi H-7100) and atomic absorption spectrometer ( $\mathrm{S}$ series) to measure density of particles inside the solutions.

\section{Results and discussion}

The prepared Ag-NPs were stable for periods of several weeks. These natural stabilizers modify Ag-Nps surface through functional group in their chemical structure (Bae et al 2011). Figure 1 represents UV-visible absorption spectra of the prepared samples after ablation of $\mathrm{Ag}$ plate in $\mathrm{Ct}, \mathrm{Gt}$, St and Wt solutions. Appearances of peaks around $400 \mathrm{~nm}$ for all samples are related to the presence of Ag-NPs in solutions. As it is clear from the figure, the maximum wavelength $\left(\lambda_{\max }\right)$ shifted to lower wavelength (from $405-403 \mathrm{~nm}$ ) by addition of Gt to Wt. According to the May theory, this blue shift is related to the reduction of particle diameter (Bohren and Huffman 1940).

The intensity of surface plasmon peak at around $400 \mathrm{~nm}$ is increased by addition of polymer to Wt. On the other hand, the intensity of peaks for St is highest in comparison to other solutions. It can be seen that using St results in highest absorbance value. The surface plasmon peak intensity is directly related to density of particles inside solutions. Therefore, from these observations we can say that concentration of Ag-NPs in St solution is highest in comparison with other polymers which we used here. On the other hand, the density of $\mathrm{Ag}$ inside the solutions is according to the following relation: $\mathrm{St}>\mathrm{Gt}>\mathrm{Ct}>\mathrm{Wt}$. This conclusion was further confirmed by measuring the density of Ag inside the solutions. The obtained amount of $\mathrm{Ag}$ for $\mathrm{Ct}, \mathrm{Gt}, \mathrm{St}$ and $\mathrm{Wt}$ are in the order of $0.40,0.32,0.11$ and $0.09 \mathrm{mg} / \mathrm{L}$.
TEM images and particle size distributions for prepared Ag-NPs at different solutions (Ct, Gt, St and Wt) are shown in figure 2. From TEM images it was found that the particle size in wt is much bigger than other solutions but minimum size was obtained for Ag-NPs in Gt. The decrease in particle size after addition of polymer to the distilled water is related to the interaction between particle and polymer molecules after formation of NPs. The mechanism of this interaction will be explained further in detail later in this article.

To compare stability of the samples we measured the UV-vis absorption spectra of the samples one month after preparation. Figure 3 shows intensity of plasmon peak at $400 \mathrm{~nm}$ for fresh and one month stored samples. The reason for decreasing absorption intensity during storage of sample is sedimentation of the colloidal particles. The sedimentation of Ag-NPs in solutions contain polymers which are less than that found in neat water, however, the minimum decrease in intensity occurred for NPs in St solution. Consequently, these data show that colloidal particles obtained in St solutions are more stable than those obtained in neat water and other polymers.

In this step we would like to explain the mechanism of absorption and capping Ag-NPs by these polymers and why the prepared NPs in St solution are more stable. NPs are formed via nucleation phase transition and crystal growth of the emitted substances from $\mathrm{Ag}$ plate during ablation process (Tsuji et al 2003). On the other hand, stabilizer passivated Ag-NPs through chemical bonding to prevent their aggregation and growth. We hypothesized that $\mathrm{Ct}, \mathrm{Gt}$ and St due to their potential for multi dentate action via their hydroxyl and amide groups, might be able to interact with Ag-NPs surface charges and act as stabilizing templates for NPs synthesis (figure 4).

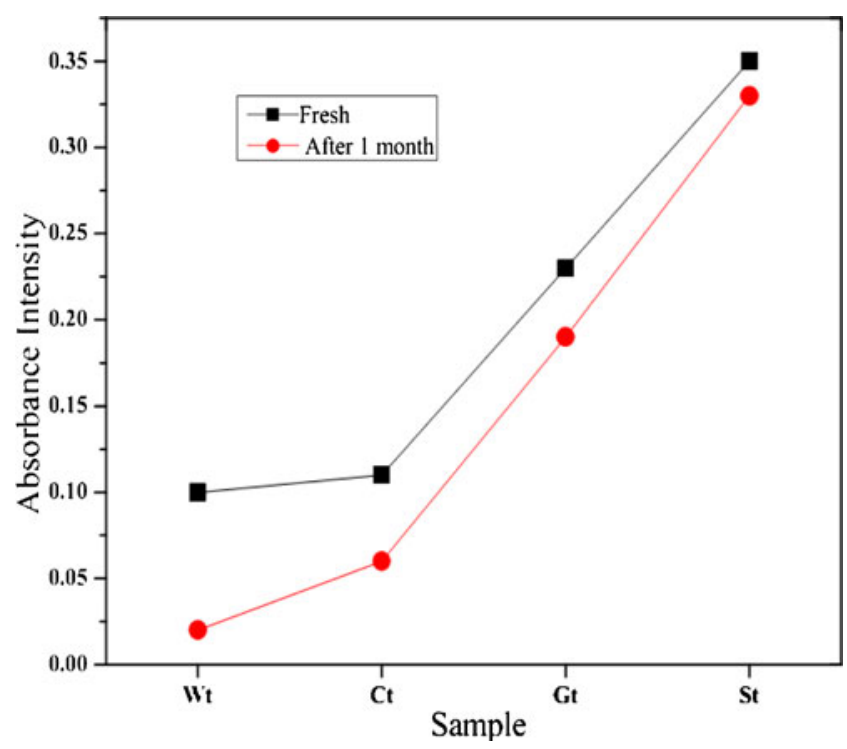

Figure 3. Absorption intensity of freshly prepared samples and after one month. 

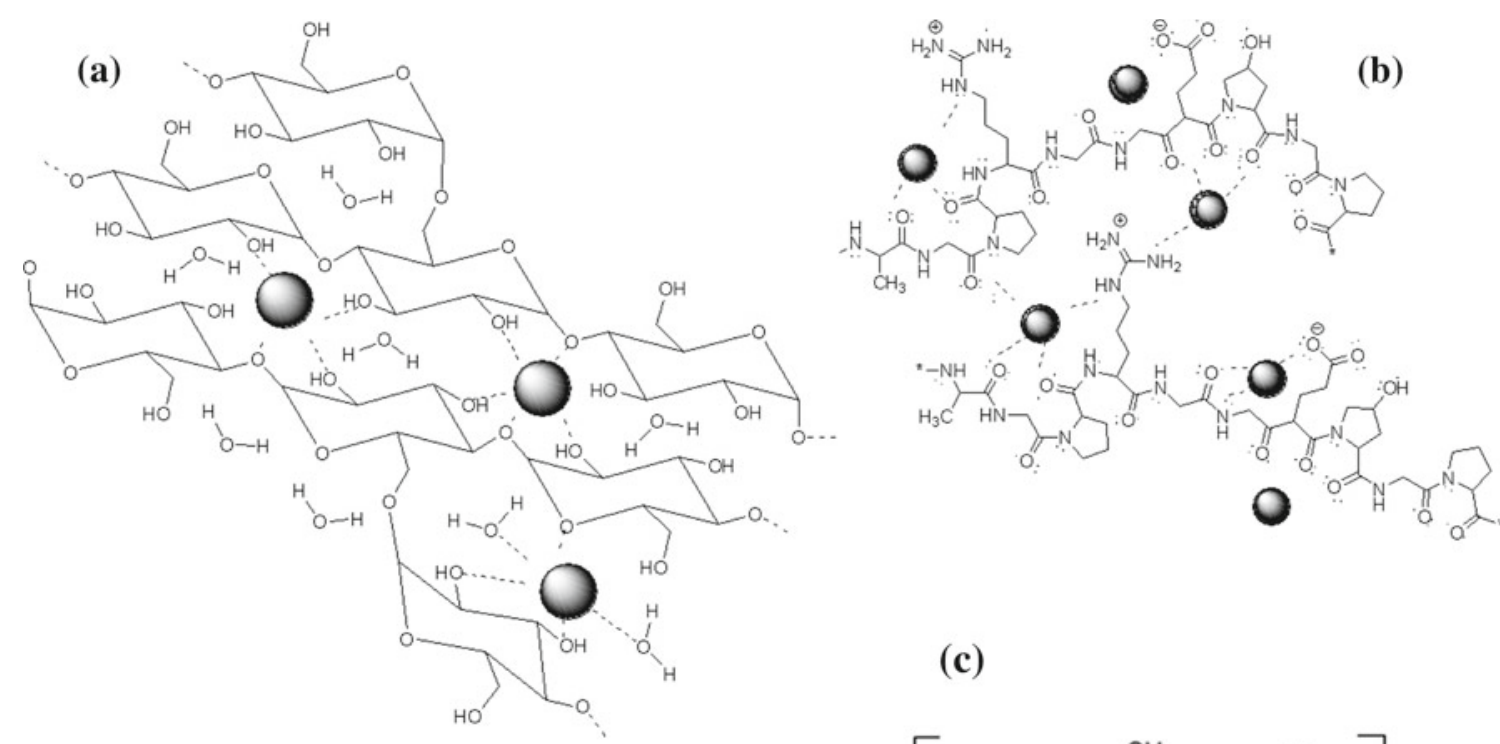

(c)

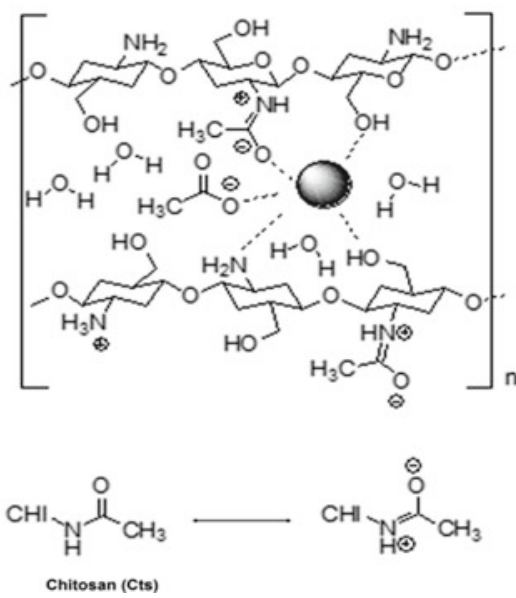

Figure 4. Mechanism of capping Ag-NPs by (a) St, (b) Gt and (c) Ct.

Upon laser ablation, many Ag atoms are produced above the laser beam contact point and $\mathrm{Ag}$ plate. Because of strong inter-atomic interactions, the produced $\mathrm{Ag}$ atoms attract each other and become aggregate. At this step, the Ag atoms interact insufficiently with stabilizer molecules. Therefore, the initial $\mathrm{Ag}$ particles are formed as a result of this inter-atomic interaction. These initial particles can grow by supplying $\mathrm{Ag}$ atoms from outside of this region through diffusion. In competition, the stabilizer molecules now can adsorb particles and limit the growth of NPs due to steric or ionic repulsion and prevent their aggregation (Mafune et al 2000). Figure 4 shows the mechanism of protecting particles from aggregation by these stabilizers.

The efficiency of St to improve stability of Ag-NPs rather than $\mathrm{Ct}$ and $\mathrm{Gt}$ can be attributed to the interaction of its hydroxyl groups and proximity of monosaccharide units in neighbouring chains that produce smaller cavity to growth of particles. On the other hand, hydroxyl group is a better chelating agent than amide group present in $\mathrm{Ct}$ and Gt because of electron withdrawing nature of carbonyl group.

\section{Conclusions}

We prepared Ag-NPs in natural polymer such as $\mathrm{Ct}, \mathrm{Gt}$ and $\mathrm{St}$ using laser ablation technique as a clean and simple method. The result showed that these natural polymers can improve formation efficiency and stability of Nps when they dissolve in Wt as a common liquid for preparation of Nps by laser ablation. On the other, a comparison between them showed that St can act more effectively than others in both aspects, increasing formation efficiency and stability. The best reason for better capping of NPs by starch is interaction of its hydroxyl groups and proximity of monosaccharide units in neighbouring chains that produce smaller cavity to growth of particles.

\section{References}

Bae C H, Nam S H and Park S M 2002 Appl. Surf. Sci. 197628

Bae E, Park H J, Park J, Yoon J, Kim Y, Choi K and Yi B J 2011 Korean Chem. Soc. 32613 
Blanco-Andujar C, Tung L D and Thanh N 2010 Ann. Rep. A106 553

Bohren C F and Huffman D R 1940 Absorption and scattering of light by small particles (Canada: John Wiley \& Sons) 57-103

Darroudi M, Ahmad M B, Zamiri R, Abdullah A H, Ibrahim N A and Shahril M S 2011 J. Alloys Compd 5091301

Mafune F, Kohno J, Takeda Y, Kondow T and Sawabe H 2000 J. Phys. Chem. B104 9111

Mafune F, Kohno J, Takeda Y, Kondow T and Sawabe H 2001 J. Phys. Chem. B105 5114

Park D K, Lee S J, Lee J H, Choi M Y and Han S W 2010 Chem. Phys. Letts $\mathbf{4 8 4} 254$

Pimpang P, Sutham W, Mangkorntong N, Mangkorntong P, Choopun S and Mai Ch 2008 J. Sci. 35250
Simakin A V, Voronov V V, Shafeev G A, Brayner R and Bozon-Verduraz F 2001 Chem. Phys. Letts 348182

Tolles W M 1996 Nanotechnology 759

Tsuji T, Kakita T and Tsuji M 2003 Appl. Surf. Sci. 206314

Tsuji T, Thang D H, Okazaki Y and Nakanishi M 2008 Appl. Surf. Sci. 2545224

Zamiri R, Azmi B Z, Naseri M G, Darroudi M, Abbastabar H and Nazarpour F K 2011a Appl. Phys. A105 225

Zamiri R, Zakaria A, Darroudi M, Sadrolhosseini A R, Husin M S, Wahab Z A and Mahdi M A 2011b Appl. Phys. $\mathbf{A 1 0 2}$ 189

Zamiri R, Zakaria A, Sadrolhosseini A R, Ahangar H A, Zaidan A W and Mahdi M A 2011c Int. J. Nanomedicine 671

Zhang G R and Xu B Q 2010 Nanoscale 22798 